\title{
Absolute Measurement of the Critical Properties of Second Sound at the Smectic-A-to-Nematic Phase Transition in 80CB
}

\section{Citation}

Fisch, M. R., L. B. Sorensen, and Peter S. Pershan. 1981. Absolute measurement of the critical properties of second sound at the smectic-A-to-nematic phase transition in 80CB. Physical Review Letters 47(1): 43-46.

\section{Published Version}

doi:10.1103/PhysRevLett.47.43

\section{Permanent link}

http://nrs.harvard.edu/urn-3:HUL.InstRepos:10361977

\section{Terms of Use}

This article was downloaded from Harvard University's DASH repository, and is made available under the terms and conditions applicable to Other Posted Material, as set forth at http:// nrs.harvard.edu/urn-3:HUL.InstRepos:dash.current.terms-of-use\#LAA

\section{Share Your Story}

The Harvard community has made this article openly available.

Please share how this access benefits you. Submit a story.

\section{Accessibility}




\title{
Absolute Measurement of the Critical Properties of Second Sound at the Smectic- $A$-to-Nematic Phase Transition in $80 \mathrm{CB}$
}

\author{
M. R. Fisch, L. B. Sorensen, and P. S. Pershan \\ Gordon McKay Laboratory, Division of Applied Sciences, Harvard University, Cambridge, Massachusetts 02138
} (Received 3 April 1981)

\begin{abstract}
Light scattered from the driven free surface of the smectic- $A$ phase of octyloxy cyanobiphenyl $(8 \mathrm{OCB})$ provides a low-frequency measurement of second sound. This yields an absolute measurement of the smectic elastic constant, $B$. There is no dispersion in either the angular or frequency dependence of $B$ for angles $35^{\circ}-83^{\circ}$ from the normal and for frequencies from $10-100 \mathrm{kHz}$. Near the smectic- $A$-to-nematic phase transition the smectic elastic constant can be described by $B=(6.03 \pm 0.05) \times 10^{7}\left(T_{N A}-T\right)^{0.32 \pm 0.015} \mathrm{dyn} / \mathrm{cm}^{2}$.
\end{abstract}

PACS numbers: 64.70.Ew, 61.30.Eb, 78.20.Hp

A principal result of the theory that de Gennes proposed in $1972^{1}$ to describe second-order smectic- $A$-to-nematic phase transitions was that the elastic constant $B$ that describes resistance to changes in the smectic layer spacing should go to zero continuously, $B \sim\left(T_{N A}-T\right)^{\phi}$. Almost all previous measurement $\mathrm{s}^{2-7}$ of the temperature dependence of $B$ have been indirect in that the quantity actually measured was $\lambda=(K / B)^{1 / 2}$. Although in many materials the Frank elastic constant, $K$, is believed to be relatively independent of temperature, direct measurements of $K$ in the smectic$A$ phase are very difficult. In contrast, second sound provides a direct measurement of the temperature dependence of $B$. There has been only one previous study of $B$ which makes use of the second sound. ${ }^{8}$

In this Letter we present the first simultaneous measurement of the frequency, wave-vector, and critical-temperature dependence of second sound near the smectic- $A$-nematic phase transition. ${ }^{9}$ From these measurements we have determined the absolute value of the smectic elastic constant, $B{ }^{10}$ By using thin samples $(\sim 150-450 \mu \mathrm{m}$ thick) of octyloxy cyanobiphenyl (8OCB), in which the top surface was a free liquid-crystal-air interface, low-frequency second sound was observed through light scattered by driven deformations of the free surface. The measurements all yield the same value of $B$ indicating the absence of any dispersion effects over a wide range of wave vectors (varying in angle by $35^{\circ}-85^{\circ}$ from the normal and in magnitude from $100-400 \mathrm{~cm}^{-1}$ ), and ranging in frequency from $10-100 \mathrm{kHz}$. The material studied, $80 \mathrm{CB}$, was chosen because of its chemical stability; it was obtained from British Drug House and used without further purification.

The experimental apparatus, shown schematically in Fig. 1, employs several techniques that were developed in other laboratories. ${ }^{11,12}$ An argon-ion laser $(\sim 30 \mathrm{~mW}, 5145 \AA)$ is weakly focused onto the free surface of the sample. The surface is driven by an alternating potential that is applied between a knife edge approximately 25 $\mu \mathrm{m}$ above the sample and a conducting substrate that makes up the bottom surface of the liquidcrystal cell. This surface was treated with the surfactant hexadecyltrimethylammonium bromide (HTAB) so that the 8OCB molecules stand normal to the glass. To obtain a flat free surface the slope of the side walls of the sample cell were adjusted to equal the contact angle, $\alpha$. The motion of the free liquid-crystal surface, in re-
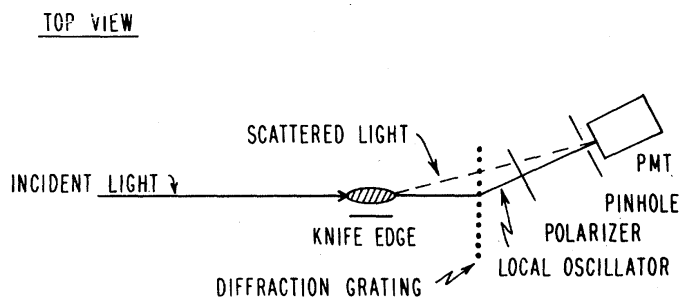

SIDE VIEW

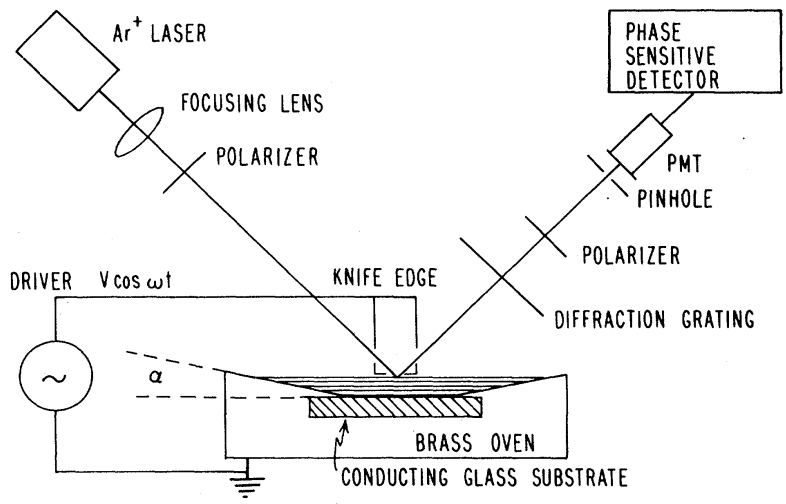

FIG. 1. Schematic of the experimental arrangement and sample cell. 

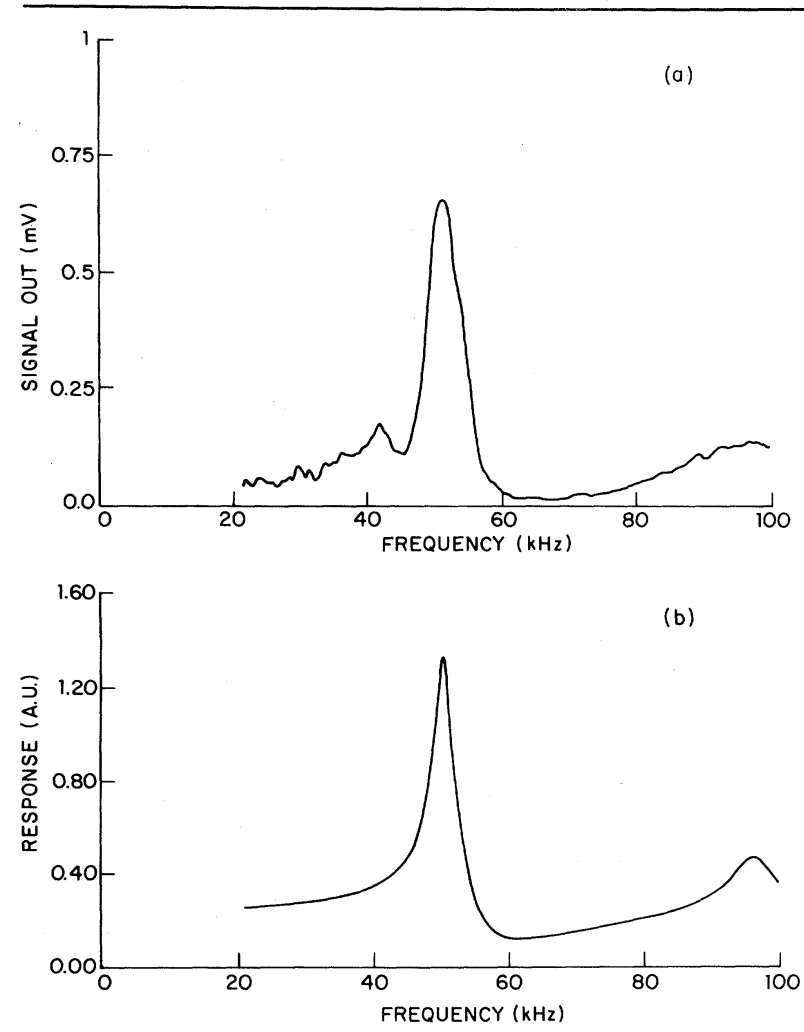

FIG. 2. (a) The experimental response as a function of frequency, for a sample with $q_{z}=\pi / 2 h=60 \mathrm{~cm}^{-1}, q_{x}$ $=125 \mathrm{~cm}^{-1}$, and $\Delta T=0.14 \mathrm{~K}$. (b) The theoretical response for $B=3.51 \times 10^{7} \mathrm{dyn} / \mathrm{cm}^{2}, \eta=0.9 \mathrm{P}$ (independent of angle), $h=262 \mu \mathrm{m}, q_{x}=125 \mathrm{~cm}^{-1}$, and $\rho=1 \mathrm{~g} / \mathrm{cm}^{3}$.

sponse to the applied voltage, scatters some of the laser light which is then mixed with a local oscillator produced by a weak diffraction grating.
Heterodyne detection at a specific diffraction maximum of the grating selects a single known component of the wave vector, $q_{x}$, parallel to the surface. The induced motion is kept small $(<0.05$ $\AA$ ) in order to minimize perturbations to the smectic layers.

The frequency and wave-vector response $\chi(\vec{q}, \omega)$ of the free surface to the applied force can be obtained by solving the hydrodynamic equations ${ }^{13}$ with the appropriate boundary conditions. To a first approximation, $\chi(\vec{q}, \omega)$ is simply given by a linear superposition of simple harmonic-oscillator responses in which the characteristic frequencies are obtained from the hydrodynamics of second sound. Specifically, for modes of the form $u(x, z)=u(z) \exp \left[i\left(\omega t-q_{x} x\right)\right]$, with $\omega \ll v_{0} q_{x}$ (where $v_{0}$ is the longitudinal sound speed), the dispersion relation becomes

$$
\begin{aligned}
& i \omega \nu_{\mathrm{eff}}\left(q_{x}^{4}+q_{z}{ }^{4}\right) \\
& +\left[(B / \rho) q_{x}{ }^{2}-\omega^{2}+i \omega \nu_{\mathrm{eff}} q_{x}{ }^{2}\right] q_{z}{ }^{2}-\omega^{2} q_{x}{ }^{2} \simeq 0,
\end{aligned}
$$

where $q_{x}$ and $q_{z}$ are the components of the wave vector in the smectic planes and normal to them, and $\nu_{\text {eff }}$ is the kinematic viscosity which is a function of the angle $\psi=\tan ^{-1}\left(q_{x} / q_{z}\right) .^{14}$ Further the modes are wave-guide-like with

$$
q_{z} \approx(\pi / 2 h)(2 m+1), \quad m=0,1,2, \ldots .
$$

The response is that of a driven harmonic oscillator with a resonant frequency that is only slightly shifted from $\omega_{0}=(B / \rho)^{1 / 2} q_{x} q_{z}\left(q_{x}{ }^{2}+q_{z}{ }^{2}\right)^{-1 / 2}$ because of viscous effects, and a full width at half maximum $\Delta \omega=2 \nu_{\text {eff }}\left(q_{x}^{2}+q_{z}{ }^{2}\right)$. The measured and predicted signals as a function of frequency are

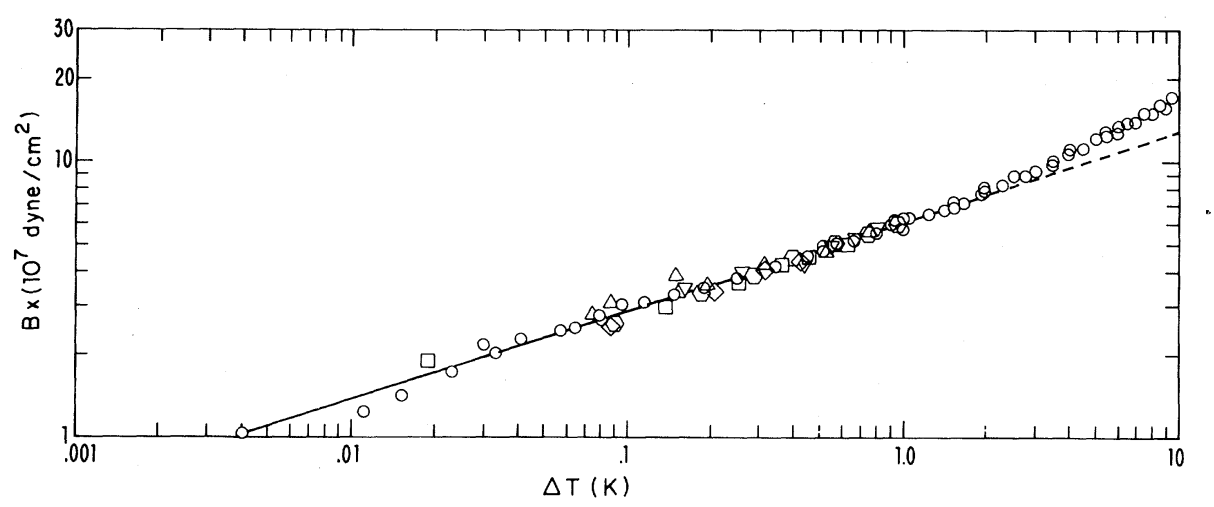

FIG. 3. $B(\Delta T)$ for different thickness samples and different scattering wave vectors. The symbols represent the different measurements: squares, $q_{z}=112 \mathrm{~cm}^{-1}, q_{x}=125 \mathrm{~cm}^{-1}$; triangles, $q_{z}=55 \mathrm{~cm}^{-1}, q_{x}=125 \mathrm{~cm}^{-1}$; diamonds, $q_{z}=55 \mathrm{~cm}^{-1}, q_{x}=250 \mathrm{~cm}^{-1}$; plusses, $q_{z}=55 \mathrm{~cm}^{-1}, q_{x}=375 \mathrm{~cm}^{-1}$; inverted triangles, $q_{z}=60 \mathrm{~cm}^{-1}, q_{x}=125 \mathrm{~cm}^{-1}$; hexagons, $q_{z}=33.4 \mathrm{~cm}^{-1}, q_{x}=125 \mathrm{~cm}^{-1}$; circles, $q_{z}=38.9 \mathrm{~cm}^{-1}, q_{x}=125 \mathrm{~cm}^{-1}$. The solid line represents the leastsquares fit to the data for $\Delta T<1.24 \mathrm{~K}$; its continuation is the broken line. 
shown in Fig. 2.

The measured $B\left(\Delta T=T_{N A}-T\right)$ for all the samples versus the respective $\Delta T$ for each of the samples are shown in Fig. 3. Although the transition temperature, $T_{N A}$, was not the same for all the samples, the $B(\Delta T)$ curve is universal. The asymptotic region, corresponding to $\Delta T$ $\leqslant 1.24 \mathrm{~K}$, was least-squares fitted to a simple power law of the form $B=B_{0}(\Delta T)^{\phi}$ yielding $B_{0}$ $=(6.03 \pm 0.05) \times 10^{7} \mathrm{dyn} / \mathrm{cm}^{2}$ and $\phi=0.32 \pm 0.015$ with $\chi^{2}=1.06$. Nelson and Toner ${ }^{15}$ recently proposed a model in which $B$ is not zero but a constant at the transition temperature. This could be described by $B=B_{0}{ }^{\prime}+B_{1}{ }^{\prime}(\Delta T)^{\phi}$. A leastsquares fit of the data in Fig. 3 to this form yields $B_{0}{ }^{\prime}=(0.15 \pm 0.43) \times 10^{7} \mathrm{dyn} / \mathrm{cm}^{2}, B_{1}{ }^{\prime}=(5.9$ $\pm 0.45) \times 10^{7} \mathrm{dyn} / \mathrm{cm}^{2}$, and $\phi=0.33 \pm 0.04$, with $\chi^{2}$ $=1.0$. Thus our data are consistent with either a simple power law or the Nelson-Toner form in which $B_{0}^{\prime} \leqslant 5 \times 10^{6} \mathrm{dyn} / \mathrm{cm}^{2}$.

A comparison between the wave-vector dependence of the results at two different temperatures and the hydrodynamic prediction is shown in Fig. 4(a). To reduce all of the data to a dimensionless form that is independent of temperature the figure shows $\Omega(\psi)=\omega_{0} /|q|\left(B_{\text {fit }}\right)^{1 / 2}$, where $\omega_{0}$ is the resonant frequency of the response, $|q|$ is the magni-

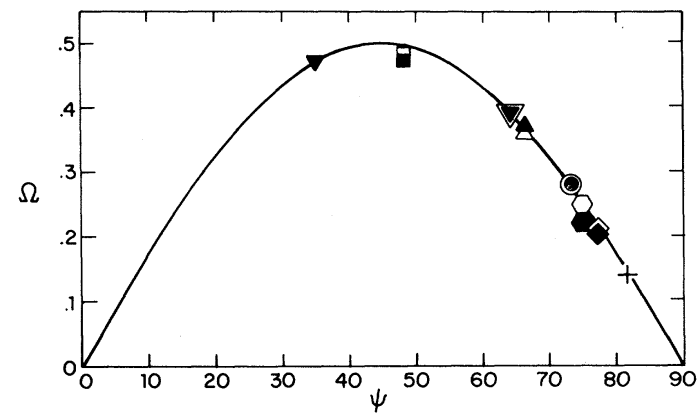

(a)

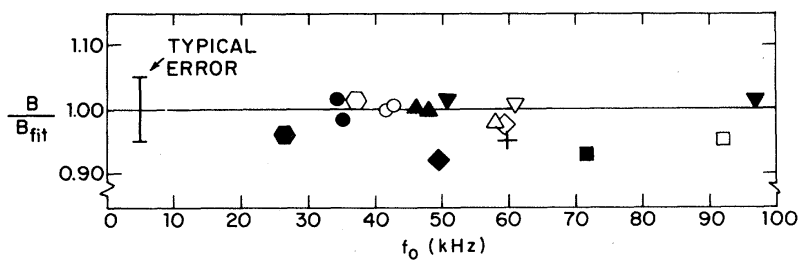

(b)

FIG. 4. (a) $\Omega(\psi)=\omega_{0} /|q|\left(B_{\text {fit }}\right)^{1 / 2}$ for two temperatures. The solid line is the hydrodynamic prediction. (b) $B /$ $B_{\text {fit }}$ vs $\omega_{0} / 2 \pi$ for the same two temperatures. The symbols represent the same measurements as Fig. 3 . The open symbols are for $\Delta T \approx 0.54 \mathrm{~K}$ and the closed symbols for $\Delta T \simeq 0.17 \mathrm{~K}$. tude of the wave vector, and $B_{\text {fit }}$ is the value of $B(\Delta T)$ that is obtained from the best fit to the data in Fig. 3. It is clear that there are no observable systematic deviations from the hydrodynamic form. For the same two temperatures, Fig. 4 (b) shows the frequency dependence of $B / B_{\text {fit }}$. Once again there are no apparent systematic deviations from the hydrodynamic prediction. There is no evidence for the angle- and frequency-dependent corrections to the second-sound speed proposed by $\mathrm{Liu}^{16}$ and Jähnig ${ }^{17}$ in the frequency range of the present experiment $(10-100 \mathrm{kHz})$. In conclusion, we have presented the first complete study of second sound near the smectic- $A$ to-nematic phase transition. There is no observable angle- or frequency-dependent dispersion in $B$ for the frequency region $10-100 \mathrm{kHz}$, and for wave vectors varying in angle $\psi$ from $35^{\circ}-83^{\circ}$. Further, we have presented the first absolute measurement of the critical properties of $B$ at low frequencies. Finally, the critical exponent describing the smectic elastic constant $B$ has been directly measured for the first time. If we assume a simple-power-law form, we obtain a value $\phi=0.32 \pm 0.015$. This can be compared with values obtained by other researchers by use of indirect techniques, which measure $\lambda=(K / B)^{1 / 2}$ rather than $B$.

In particular, for $80 \mathrm{CB}$, Lister et al. ${ }^{2}$ obtained $\phi=0.26 \pm 0.08$. For 4 -cyano-4' - octylbiphenyl (8CB), Davidov et al. ${ }^{3}$ found $\phi=0.26 \pm 0.06$, for $\mathrm{N}-p$-cyanobenzylidene- $p$-octyloxyanilene (CBOOA), Birecki et al. ${ }^{4}$ and Ribotta ${ }^{5}$ measured $\phi=0.33$ \pm 0.05 and $\mathrm{Clark}^{6}$ found $\phi=0.36 \pm 0.14$, and for butoxybenzilidene octylaniline $(40.8)$ von Känel and Litster ${ }^{7}$ obtained $\phi=0.32 \pm 0.03$. For all of these smectic- $A$-to-nematic transitions, $\phi$ is close to a value of $\frac{1}{3}$. Further measurements are required to determine whether there is a universal exponent for the smectic elastic constant.

One of us (M.R.F.) is a recipient of an IBM Predoctorial Fellowship. This work was supported by the National Science Foundation under Grant No. DMR-77-24295 and by the Joint Services Electronics Program (U. S. Army, Navy, and Air Force) under Grant No. N00014-75-C-0648.

\footnotetext{
${ }^{1}$ P. G. de Gennes, Solid State Commun. 10, 753 (1972). ${ }^{2}$ J. D. Litster, J. Als-Nielsen, R. J. Birgeneau, S. S. Dana, D. Davidov, F. Garcia-Goldberg, M. Kaplan, C. R. Safinya, and R. Schaetzing, J. Phys. (Paris), Col-
} 
loq. 40, C3-339 (1979).

${ }^{3}$ D. Davidov, C. R. Sanfinya, M. Kaplan, S. S. Dana, R. Schaetzing, R. J. Birgeneau, and J. D. Litster, Phys. Rev. B 19, 1637 (1979).

${ }^{4}$ H. Birecki, R. Schaetzing, F. Rondelez, and J. D. Litster, Phys. Rev. Lett. 36, 1376 (1976).

${ }^{5}$ R. Ribotta, C. R. Acad. Sci. 279B, 295 (1974).

${ }^{6}$ Noel A. Clark, Phys. Rev. A 14 , 1551 (1976).

${ }^{7}$ H. von Känel and J. D. Litster, Phys. Rev. A $\underline{23}$, 3251 (1981) .

${ }^{8}$ The temperature dependence of $B$ for $8 \mathrm{CB}$ has been measured by L. Ricard and J. Prost, J. Phys. (Paris), Colloq. 40, C3-83 (1979).

${ }^{9}$ After completing this work we became aware of a similar measurement by L. Ricard and J. Prost (to be published).

${ }^{10}$ Different definitions of the smectic elastic constant $B$ are possible, depending on whether it is at constant pressure or constant density I see, for example, P. C. Martin, O. Parodi, and P. S. Pershan, Phys. Rev. A $\underline{6}$,
2401 (1972)]. The present measurements actually determine $\widetilde{B}=B / \rho$, where $B$ is the elastic constant at constant density and entropy, and $\rho$ is the density. The density is close to unity and since it has no significant temperature variation near $T_{N A}$, we have taken $\widetilde{B}=B$. IF. R. Bouchet and P. E. Cladis, Mol. Cryst. Liquid Cryst. 64, 8L (1980).]

${ }^{11}$ C. H. Sohl, K. Miyano, and J. B. Ketterson, Rev. Sci. Instrum. $\underline{49}, 1464$ (1978).

${ }^{12}$ S. Hård, Y. Hammerius, and D. Nilsson, J. Appl. Phys. 47, 2433 (1976).

${ }^{13}$ Martin, Parodi, and Pershan, Ref. 10.

${ }^{14}$ The viscosity that describes the second-sound mode in the smectic $-A$ is given by $\nu_{\text {eff }}=\rho^{-1}\left[\eta_{44}+\left(\eta_{11}+\eta_{33}-2 \eta_{13}\right.\right.$ $\left.-4 \eta_{44}\right) \cos ^{2} \psi \sin ^{2} \psi$ ] [K. Miyano and J. B. Ketterson, Physical Acoustics Principles and Methods (Academic, New York, 1979), Vol. 14, p. 93.

${ }^{15}$ David R. Nelson and John Toner, to be published.

${ }^{16}$ Mario Liu, Phys. Rev. A 19, 2090 (1979).

${ }^{17}$ F. Jähnig, J. Phys. (Paris) 36, 315 (1975).

\title{
Direct Nuclear-Magnetic-Resonance Measurements of Biaxiality in the Cholesteric Liquid Crystalline Phase
}

\author{
Zvi Yaniv, Nuno A. P. Vaz, ${ }^{(a)}$ Giuseppe Chidichimo, ${ }^{(b)}$ and J. William Doane \\ Department of Physics and Liquid Crystal Institute, Kent State University, Kent, Ohio 44242 \\ (Received 7 April 1981)
}

\begin{abstract}
We report what we believe is the first experimental measurement of biaxiality in a cholesteric phase. This observation is made in a selectively deuterated nematic that was twisted by the addition of a chiral compound. A theoretically predicted biaxial order parameter (measured in terms of an asymmetry in the time-averaged deuterium quadrupole interaction) was found to be $\sim 10^{-3}$ for a pitch $\sim 3 \mu \mathrm{m}$ and to increase both with decreasing pitch and as the isotropic phase is approached.
\end{abstract}

PACS numbers: 64.70.Ew, 61.30.Gd

The possibility of observing biaxial molecular order in a twisted nematic has been a topic of discussion for several years. ${ }^{1}$ The nematic liquid crystal is normally uniaxial, but when it is twisted the cylindrical symmetry is broken by the helical twist defined by the pitch axis and biaxial ordering, though perhaps very weak, has been expected in such systems. Several theoretical investigations have regarded biaxiality as an important feature of cholesteric $\mathrm{s}^{2-4}$ and of the blue phase ${ }^{5,6}$ which often intercede between the cholesteric and the isotropic phase. In an experiment on light scattering ${ }^{7}$ the data could be interpreted as a result of competing fluctuations of both uniaxial and biaxial order parameters, but no values of the biaxial order parameters were reported. On the other hand, other optical experiments ${ }^{1}$ have not revealed any observable biaxiali- ty. Nevertheless, theory has been encouraging for further experimental study with some authors suggesting the use of NMR to observe this feature..$^{3,5}$

In this paper we report a direct deuterium NMR measurement of biaxial order in a nematic that has been twisted by an optically active compound added to the sample. The nematic material is a binary mixture of $75 \mathrm{wt} . \%$ 4-methoxybenzylidene$4^{\prime}-n$-butylaniline (MBBA) and $25 \mathrm{wt} . \% 4-n$-butyloxybenzylidene- $4^{\prime}-n$-heptyl $-d_{4}$-analine $\left(4.07-d_{4}\right)$ selectively deuterated on one aromatic ring. To this material was added various concentrations of chiral 4-cyano-4' - (2-methyl) butylbiphenyl (CB-15) to yield the twisted structure.

In the untwisted material, the nematic aligns in the magnetic field yielding the typical deuterium spectrum illustrated in Fig. 1(a). Of interest in 